\title{
Mitteilungen aus dem Laboratorium für Geophysik der Carnegie Institution in Washington. 5. \\ Schmelzpunktsbestimmungen.
}

\author{
Von \\ Walter P. White. ${ }^{1}$ \\ Mit 4 Figuren im Text.
}

Einleitung.

Die neueren grofsen Fortschritte in der Pyrometrie sowie die Entwickelung der elektrischen Öfen haben viele physikalische und physıkochemische Bestimmungen bei hohen Temperaturen fast so leicht gemacht und ihnen einen solchen Grad von Sicherheit verliehen, wie man bisher nur bei gewöhnlichen Temperaturen erreichen konnte. Die geeignete spezielle Technik, die sich erst in der letzten Zeit entwickelt hat, ist aber auch jetzt noch nicht allgemein bekannt, und auch die Wichtigkeit des ganz neuen und fruchtbaren Forschungsfeldes bei hohen Temperaturen findet nur allmählich gerechte Würdigung. Es scheint deswegen ratsam zu sein, die im geophysikalischen Laboratorium ausgearbeiteten Verfahren von Zeit zu Zeit zur allgemeinen Information mitzuteilen, abgesehen von ihrer unmittelbaren Anwendung auf unsere eigenen Arbeiten. So sind bereits zwei besondere Veröffentlichungen erfolgt, in denen die Herstellung von Öfen und die Messung der Temperatur bis $1600^{\circ}$ beschrieben wird. ${ }^{2}$ Die folgenden beiden Arbeiten beschäftigen sich mit der Anwendung dieser Messungen auf solche Methoden der physikochemischen thermischen Analyse, die jetzt, nachdem sie an besten durch ihre Aufschlüsse über die Konstitution von Metalllegierungen bekannt geworden sind, mit

1 Aus dem Amer. Journ. Science 28 (1909), 453 ins Deutsche übertragen von I. KOPPEL-Berlin.

${ }^{2}$ Day und Allen, Phys. Rev. 19 (1904), 177. - W. P. White Phys. Rev. 25 (1907), 334. Andere Mitteilungen aus diesem Laboratorium, die gelegentlich die Methodik behandeln, werden in dieser Beziebung durch die vorliegende Arbeit zusammengefalst und ergänzt, allerdings nur soweit Schmelzpunktsbestimmungen bis $1600^{\circ} \mathrm{C}$ in Frage kommen.

z. anorg. Chem. Bd. 69. 
gleichem Erfolge auf Mineralien und älnliche Verbindungen angewandt werden. Die erste Arbeit behandelt die Schmelzpunktserscheinungen im allgemeinen, und die darin enthaltenen. Schlüsse sind nicht auf das Gebiet hoher Temperaturen beschränkt; die zweite beschreibt die Ofentechnik, die erforderlich ist, um bis $1600^{\circ} \mathrm{C}$ die in der ersten dargelegten Bedingungen herzustellen.

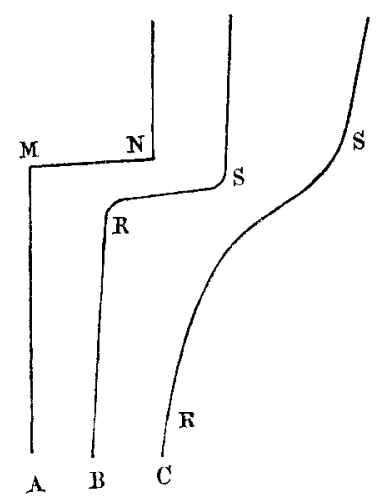

Fig. 1. Schmelzkurven; $A$ ideal; $B$ u. $C$ Kurven für Silber u. Anorthit nach Day u. Alten, "Isomorphismus $u$. thermische Eigenschaften der Feldspate."

Der hervorragende Wert von schmelzendem Kis als Bezugstemperatur hat die grofse Konstanz des idealen Schmelzpunktes und seine Unabhängigkeit ron der äufseren Temperatur bekannt gemacht. Die gröfsere Mehrzahl der tatsächlich ausgeführten Schmelzpunktsbestimmungen zeigt aber nicht diese ideale Konstanz, und weist eher ein Schmelzintervall als einen Punkt auf. Wenn die Temperatur-Zeitkurve $A$ (Fig. 1) ein ideales Schmeizen bei gleichförmiger Wärmezufuhr darstellt, wobei $M N$ das Intervall konstanter Temperatur bedeutet, so ähneln die bei Substanzen von hohem Schmelzpunkt erhaltenen Resultate mehr den geneigten Kurven $B$ und $C$, bei denen das Intervall $R S$ bis $60^{\circ}$ betragen kann.

Wenn die Schmelzpunktskurve schräg verläuft, so liegt in gröfserem Umfang die Möglichkeit für zufällige und systematische Fehler vor. Bei unseren eigenen Arbeiten waren diese, wenn man die hohen Temperaturen in Betracht zieht, nicht ernsthaft, und lange Zeit waren sie viel weniger unsicher als die extrapolierte Temperaturskala selbst. Die grölste Abweichung bei einer Anzahl Bestimmungen desselben Punktes hat nur selten $3^{0}$ erreicht. ${ }^{1}$

Aber andere Beobachter haben viel gröfsere und bisweilen verwirrende Unregelmälsigkeiten gefunden und daher den Wert der thermischen (Frankenheim-) Methode zur Schmelzpunktsbestimmung

1 Der Klarheit wegen mag sogleich betont werdèn, dafs die Fehler, die durch diese Untersuchung vermindert werden sollen, etwa $5^{\circ}$ oder weniger betragen, wie sie bei den regelmälsigen Arbeiten in unserem Laboratorium iiber Silikatschmelzen vorkommen. Mit den systematischen Differenzen von $00^{\circ}$ oder $200^{\circ}$, die bisweilen in der Literatur auftreten, lat diese Arbeit nichts zu tun. 
in Frage gezogen. Überdies waren die Ursachen des geneigten Verlaufs dieser Schmelzpunktskurven an und für sich von Interesse; und schliefslich fand sich bei der Untersuchung einiger Pyroxene ein Problem, das viel genauere vergleichende Messungen erforderte, als wir erhalten hatten. Es wurde demnach eine allgemeine Untersuchung über die thermische Methode zur Bestimmung von Schmelz-

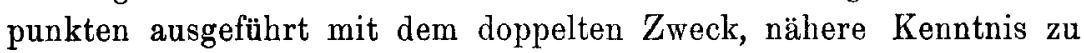
erhalten von den Eigenschaften der Materie in der Nähe der Schmelztemperatur, und unsere eigene Technik zu verbessern. Das Resultat war, da[s die Übereinstimmung bei unseren Messungen an Silikaten um ungefähr das Fünffache vergrölsert wurde, ${ }^{1}$ wobei die Versuchstechnik auch gleichzeitig erleichtert wurde. Auch ein Einblick in die obwaltenden Beziehungen liefs sich erzielen, wodurch verschiedene, bisher sehr verwirrende Fragen aufgeklärt wurden, und man fand einen Weg, die Genauigkeit noch weiter zu steigern, wenn dies notwendig erscheinen sollte.

Eine Anzahl von organischen und anorganischen Stoffen, deren Schmelzpunkte zwischen $0^{\circ}$ und $1400^{\circ}$ liegen, wurden unter verschiedenen Bedingungen untersucht. Die meisten dieser Versuche dienten zur Prüfung von Hypothesen; sie brauchen deswegen nicht im einzelnen beschrieben zu werden. Die Schlüsse, zu denen sie führten, werden vielleicht gleichfalls gewinnen, wenn man sie in anderer Reihenfolge darstellt, als sie sich aus den Untersuchungen ergaben.

Um die Gedanken zu fixieren, ist es vielleicht ron Vorteil, zuerst den allgemeinen Plan der experimentellen Anordnung bei unserer regelmäfsigen Arbeit mitzuteilen, auf die sich diese vorliegende Untersuchung direkt bezieht. Die Substanz, deren Schmelzpunkt bestimmt werden soll, befindet sich in dem Tiegel (Fig. 2). In der Mitte der Beschickung befindet sich das Thermometer, das in unserem Fall stets ein Thermoelement irgendwelcher Art ist. Dieses wird bisweilen ohne Umhüllung benutzt, bisweilen aber von einem Porzellan- oder Platinmantel umgeben, wie hier dargestellt ist. Der Tiegel wird in einem elektrischen Widerstandsofen erhitzt, für den eine Akkumulatorenbatterie einen sehr regelmälsigen Strom liefert. Ein Kontrollelement $C$ zeigt die Ofentemperaturen an und gestattet, wenn erforderlich, dessen Regulierung.

1 Diese Verbesserung ist bereits dargelegt worden in einer Arbeit über Diopsid und seine Beziehungen zu Calcium- und Magnesiummetasilikat, Amer. Journ. Sci. 27 (1909). 4. Deutsche Übersetzung für die Z. anorg. Chem. in Vorbereitung. 
Es ist den Forschern auf diesem Gebiet eine bekannte Tatsache, dafs Erstarrungspunkte oftmals schärfer sind und viel besser übereinstimmen als Schmelzpunkte. Die Ursachen dieses Unterschieds spielen in dieser Arbeit eine bedeutende Rolle. Die Folge dieser Erscheinung ist, dafs Erstarrungspunktsbestimmungen im allgemeinen vorgezogen werden; und der grölste Teil

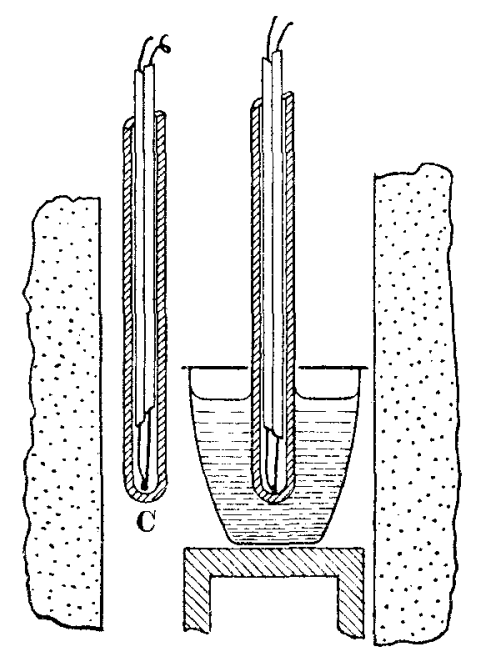

Fig. 2. Schnitt durch den gewöhnlichen Schmelzpunktsapparat. (Halbe Gröfse.) $C$, Kontrollelement. der Literatur über diesen Gegenstand bezieht sich auf Beobachtungen mit fallender Ofentemperatur. Erstarrungspunkte jedoch sind unsicher oder nutzlos bei Substanzen mit ausgesprochener Unterkühlung oder träger Kristallisation. Da dies aber bei Silikaten der gewöbnliche Fall ist, so benutzen wir trotz der gröfseren experimentellen Schwierigkeiten bei unseren Arbeiten über Silikate nur den gewöhnlichen Schmelzpunkt. Und mit diesem haben wir hauptsächlich in der vorliegenden Mitteilung zu tun. Die besonderen Nachteile der Schmelzkurve könnten ohne Zweifel durch Rühren in weitem Malse überwunden werden; aber ein wirksames Rühren ist in vielen Fällen schwierig, und in den übrigen Fällen unmöglich, so dals es niemals versucht worden ist.

Die wesentlichen Ursachen geneigter Schmelzkurven scheinen die folgenden zu sein:

A. Primäre, d. h. durch die Substanz selbst bedingte Ursachen.

I. Zeitverzögerung beim Schmelzen (bei sehr zähen Substanzen).

II. Gegenwart von Verunreinigungen.

B. Sekundäre Ursachen, die dadurch bedingt sind, dafs der Apparat nicht genan das Verhalten der Stoffe wiedergibt.

III. Inkonstante Wärmezuführung.

IV. Der, normale Temperaturgradient zwischen der Aufsenseite und dem Innern der schmelzenden Beschickung.

V. Zufällige Unregelmälsigkeiten der Temperaturverteilung.

VI. Wärmezuflufs durch das Thermoelement. 
VII. Elektrisches Leitvermögen der Schmelze, wenn das Thermoelement ungeschützt verwendet wird.

VIII. Inhomogenität des Thermoelementes.

IX: Ungleichförmiges Kristallisieren (Saigern) der Beschickung.

X. Strahlung durch die schmelzende Substanz.

I. Zähigkeit. DaY und ALLEN ${ }^{1}$ entdeckten, dafs Albit und Orthoklas eine Art Hysteresis beim Schmelzen zeigen, und diese Erscheinung hat sich seitdem auch beim Quarz gefunden. Sie steht in Verbindung mit der grofsen Zähigkeit der geschmolzenen Substanzen in der Nähe ihres Schmelzpunktes. Die Zustandsänderung tritt allmählich ein, selbst bei Temperaturen, die viele Grade $\left(100^{\circ}\right.$ oder mehr) oberhalb des Punktes liegen, bei dem sie in hinreichend langer Zeit gleichfalls bereits stattfände. Theoretisch mürste diese Erscheinung wahrscheinlich als charakteristisch bei allen Substanzen beobachtet werden, obwohl sie natürlich zu klein ist, um sich bei den meisten bemerklich zu machen. Dafs sie ganz allgemein bei den Silikaten sehr merkbar würde, schien dagegen nicht unwahrscheinlich zu sein, aber dies ist nicht der Fall. Obwohl beträchtliche Teile einer Albit- oder Orthoklasbeschickung ungeschmolzen bleiben können, wenn sie auch mehrere Stunden einer Temperatur ausgesetzt werden, die $150^{\circ}$ oder mehr über dem Punkte liegt, wo das Schmelzen beginnt, fand sich doch z. B. Diopsid, als man ihn dreimal mit einer von $4-13^{\circ}$ pro Minute wechselnden Geschwindigkeit erhitzte, Resultate zeigte, die auf $0.1^{0}$ übereinstimmten. (Das Unterscheidungsmerkmal für diese Erscheinung ist natürlich die Veränderlichkeit der Schmelztemperatur mit der Erhitzungsgeschwindigkeit). Hysteresis beim Schmelzen ist demnach nur eine gelegentliche Ursache der Neigung der Schmelzkurven. ${ }^{2}$

II. Verunreinigungen. Der Einflufs von Verunreinigungen auf die Verminderung der Schärfe des Schmelzpunktes ist ziemlich bekannt, besonders bei den organischen Chemikern. Die Art des Einflusses auf die Temperatur-Zeitkurve scheint aber nur wenig studiert $\mathrm{zu}$ sein und seine Wichtigkeit und Gröfse sind oftmals unterschätzt worden. Er ist in der Tat eine direkte Folge der Schmelzpunktserniedrigung durch die Verunreinigung, und aus dem Gesetz dieser Erniedrigung kann ein Ausdruck dafür abgeleitet

${ }^{1}$ Isomorphismus und thermische Eigenschaften der Feldspate. Publ. Carnegie Inst. Washington Nr. 31, p. 50-54; Amer. Journ. Sei. [4] 19 (1905), 119.

${ }^{2}$ Ist die Hysteresis tatsächlich beträchtlich, so mufs natürlich die Methode zur Bestimmung des Schmelzpunktes wesentlich geändert werden. Siehe die nächste Mitteilung, S. 343. 
werden. Dieser nimmt eine sehr einfache Form an für den sehr gewöhnlichen und wichtigen Fall, dals die Erniedrigung des Schmelzpunktes der Menge der Verunreinigung proportional ist. Eine Besprechung dieses Falles wird den gewöhnlichen Charakter dieses wichtigen Effektes klar legen.

Um eine Schmelzpunktskurve zu definieren, sind zwei Grölsen notwendig und im allgemeinen auch hinreichend: die Temperaturerhöhung $(d \theta)$ und die Wärmemenge $(d Q)$ die erforderlich ist, um sie hervorzubringen. In der Tat ist die wahre Schmelzkurve nur der graphische Ausdruck der Beziehung dieser beiden Grölsen, d. h. des Quotienten $\frac{d Q}{d \theta}$. Wenn man die Temperaturerhöhung gegen die Zeit aufzeichnet, so dient die Zeit in Wirklichkeit nur als angenähertes Mals der zugeführten Wärme. $\frac{d Q}{d \theta}$ ist aber nach Definition die spezifische Wärme. ${ }^{1}$ Demnach kann man in der Regel einen schmelzenden Körper vollständig thermisch beschreiben, indem man ihn als einen Körper von aufserordentlich wechselnder spezifischer Wärme behandelt und dies ist oft der einfachste und leichteste Weg der Darstellung.

Der Einflufs einer Verunreinigung auf die Schmelzkurve ist demnach ibr Einflufs auf den Quotienten $\frac{d Q}{d \theta}$, den man in der folgenden Weise für den Fall findet, daf's die Schmelzpunktserniedrigung der Menge der vorhandenen Verunreinigung proportional ist.

Wir wollen den Schmelzpunkt der vollkommen reinen Substanz als Temperatur 0 annehmen (die Temperaturen während des Schmelzens sind dann negativ und nebmen numerisch ab, wenn die Substanz wärmer wird); $\theta_{o}$ sei die Frniedrigung des Schmelzpunktes in dem gegebenen Fall. Dann ist $\theta_{o}$ die Temperatur, bei der die vorhandene Verunreinigung gerade die hinreichende Konzentration hat, um die ganze Masse des Lösungsmittels in den flüssigen Zustand zu bringen. Man lasse sodann die Hälfte des Lösungsmittels durch Erniedrigung der Temperatur auskristallisieren. Die Konzentration der Verunreinigung ist jetzt doppelt so grols wie vorher und die Erniedrigung der Temperatur nach der Annahme

$1 \frac{d Q}{d \theta}$ kann natürlich auch so gemessen werden, dafs es der Wärmekapazität gleich ist. $\mathrm{Da}$ beide Gröfsen für dieselbe Beschickung einander proportional sind, so ist die Unterscheidung hier nicht von Wichtigkeit. 
gleichfalls doppelt, also gleich $2 \theta_{o}$; in ähnlicher Weise wird ein Drittel des Lösungsmittels flüssig bleiben bei $3 \theta_{0^{\circ}}$. Wenn nun $A$ der Bruchteil des in flüssiger Form vorhandenen Lösungsmittels ist, so wird $A \propto \frac{1}{\theta}$; dies kann man schreiben $A=\frac{K}{\theta}$, wo $K$ eine zu bestimmende Konstante ist.

Nun ist die Geschwindigkeit der Wärmeabsorption beim Schmelzen, d. h. der Teil von $\frac{d Q}{d \theta}$, der für das Schmelzen erforderlich ist, proportional der durch die Einheit der Temperatursteigerung geschmolzenen Menge, d. h. proportional $\frac{d A}{d \theta}$ oder gleich $m \frac{d A}{d \theta}$, wo $m$ nur ein Proportionalitätsfaktor ist. Aber $\frac{d A}{d \theta}=-\frac{K}{\theta^{2}}$, und da das Integral ron $m \frac{d A}{d \theta}$ d. h. das Integral von $m \frac{K}{\theta^{2}}$ von $\theta_{o}$ bis $\infty$ der Schmelzwärme $L$ gleich sein muls, so ist $m K$ gleich $L \theta_{\circ}$. Wenn demnach $S$ für die wahre spezifische Wärme bei irgend einer Temperatur gesetzt wird, so ist die totale virtuelle oder scheinbare spezifische Wärme $\sum$ für Temperaturen unterhalb und nicht zu weit von $\theta_{o}$.

$$
\Sigma=S+\frac{L \theta_{o}}{\theta^{2}}
$$

Durch den gröfsten Teil des Intervalles, wo die Gleichung benutzt werden kann, ist $S$ verhältnismälsig so klein, dals es für gewöhnlich vernachlässigt werden kann, und die Funktion kann einfach geschrieben werden.

$$
\Sigma=\frac{L \theta_{o}}{\theta^{2}}
$$

Die Form der sich ergebenden Schmelzkurre kann nunmehr erhalten werden, indem man $\frac{d Q}{d \theta}$ für $\sum$ setzt, und von $\theta_{o}$ bis $\theta$ integriert, wobei man erhält

$$
[Q]_{\theta_{o}}^{\theta}=\left(S+\frac{L}{\theta}\right)\left(\theta-\theta_{o}\right)
$$

woraus $Q$ in Ausdrücken von $\theta$ berechnet werden kann. Wenn (2) anstatt (1) benutzt wird, erhält man $Q=L-\frac{L \theta_{o}}{\theta}$ woraus dann

$$
\theta=\frac{L \theta_{0}}{L-Q} \text { wird. }
$$


Hieraus kann $\Theta$ durch $Q$ dargestellt werden. Hier wird $\Theta$ noch gemessen nach unten rom wahren Schmelzpunkt und $Q$ ist in der Tat die abgegebene Wärmemenge, wenn der Körper erstarrt. Aber natürlich gibt dieser Ausdruck die Form der Kurve so gut wie irgend ein anderer, und dabei ist er einfacher als ein Ausdruck mit positiven Temperaturen und Wärmemengen sein würde.

Eine derartige Kurve ist die Kurve von Fig. 3.

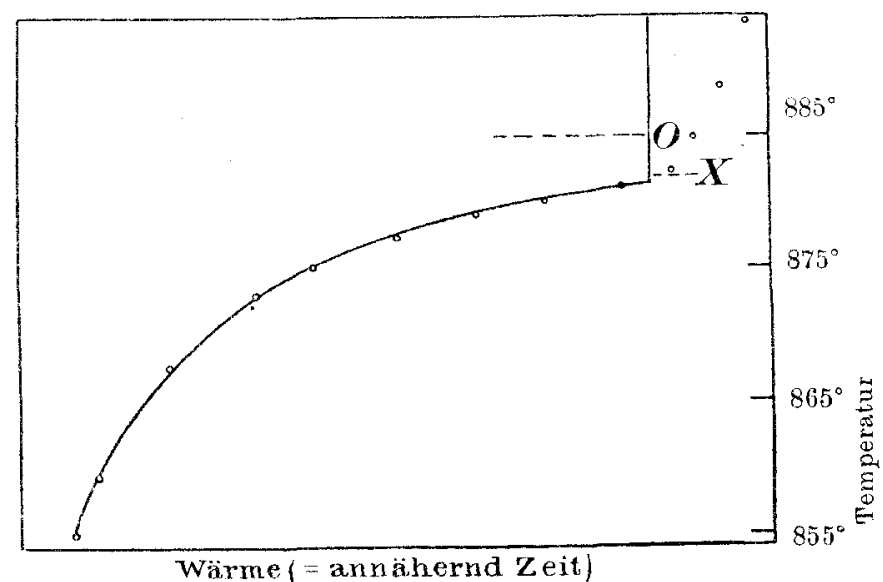

Fig. 3. Typische Schmelzkurve. Die Punkte sind einer an $\mathrm{Na}_{2} \mathrm{SO}_{4}$ mit $1 / 3 \%$ $\mathrm{NaCl}$ aufgenommenen Kurve entnommen. $X-O$ Erni drigung des Schmelzpunktes unter den der reinen Substanz. Die Kurve wurde berechnet nach Formel 3 für die Depression $X-O$. Daten finden sich S. 344.

Die kleinen Kreise zeigen eine in der Nähe von $900^{\circ}$ tatsächlich erhaltene Kurve in einem Falle, wo alle anderen Ursachen von Abweichungen praktisch ausgeschlossen waren.

Die Kenntnis der Rolle, die Verunreinigungen spielen, verändert gänzlich die gewöhnliche Auffassung der Schmelzpunktserscheinungen. Der Beobachter hat nicht mit einer konstanten Temperatur zu tun, sondern mit einer von Anfang bis zum Ende veränderlichen. ${ }^{1}$ Der Verlauf der Erscheinungen ist durchaus nicht unabhängig von der Ofentemperatur, sondern folgt ihr sehr

1 Die Tatsache, dafs es sich in Wirklichkeit um ein Schmelzintervall und nicht um einen Schmelzpunkt handelt, ist von einigen Beobachtern erkannt worden, die regelmälsig das obere und untere Ende des Intervalles bestimmen. Es ist nach der obigen Auseinandersetzung jedoch klar, dafs die Bezeichnung einer unteren Grenze ganz willkürlich ist. 
nahe und verzeichnet alle ihre Schwankungen. Einige Folgerungen aus dieser Anschaung bedürfen noch einer näheren Besprechung.

1. Die Genauigkeit der thermischen Methode. Die Neigungen der thermischen Kurven sind von mehreren Beobachtern als Beweis für die Ungenauigkeit und Unzuverlässigkeit der thermischen Methode angeführt worden. Das trifft nicht zu, denn sie sind eher gute Anzeigen für eine getreue Anpassung an die Erscheinungen. Wenn ein Verfahren angegeben werden könnte, das bei gewöhnlichen Substanzen vollkommen scharfe Punkte lieferte, so würde gerade dadurch der Beweis erbracht sein, dals es die Tatsachen entstellt und demnach unzuverlässig ist. Und während zum Beispiel das Verfahren zur Bestimmung von Schmelztemperaturen durch Beobachtung der Änderungen des Aussehens der Substanz das allmähliche Fortschreiten des Schmelzens zeigt, sind 'seine Resultate viel weiter vom Quantitativen entfernt. Es besteht kein bestimmter Zusammenhang zwischen der geschmolzenen Materialmenge und der äufseren Erscheinung der Beschickung. Die thermische Methode allein gibt bei hohen Temperaturen die Möglichkeit, genau den Schmelzprozels rom Anfang bis zum Ende darzustellen.

2. Änderung der Neigung mit der Temperatur. Entsprechend der bekannten Formel $\Delta=\frac{0.02 \theta^{2}}{L}$ ist die Schmelzpunkterniedrigung $\Delta$ proportional dem Quadrat der absoluten Temperatur und demnach (siehe z. B. Gleichung 3) muls die Neigung der Schmelzkurve sich in derselben Weise ändern. Daher stammen die grofsen Wirkungen durch Verunreinigungen bei hoher Temperatur, die bei niedriger Temperatur ganz zu vernachlässigen wäre. Dies kennzeichnet ungefähr der folgende Fall: $0.1 \%$ einer Verunreinigung vom Molekulargewicht 80, etwa $\mathrm{CaF}_{2}$, welches auf die doppelte Anzahl von Molekeln dissoziitiert ist, würde nach der Formel den normalen Schmelzpunkt von Diopsid (13919) um $1.3^{0}$ erniedrigen und würde eine scheinbare Verdoppelung der spezifischen Wärme $20^{\circ}$ darunter verursachen, während, wenn Gleichung (1) genau gültig wäre, der Beginn des Schmelzens noch leicht $50^{\circ}$ tiefer bemerkbar sein mülste. Eine ähnliche Verunreinigung würde den Schmelzpunkt des Eises nur um $0.05^{\circ}$ erniedrigen, und die Verdoppelung der scheinbaren spezifischen Wärme würde bereits $3^{0}$ unter der Schmelztemperatur eintreten.

Diese Tatsachen beziehen sich direkt auf die Frage nach dem Wert von Schmelzpunktsbestimmungen bei natürlichen Mineralien. 
Die Verunreinigungen natürlicher Mineralien belaufen sich nur in wenigen Fällen auf einige T'eile in 1000; gewöhnlich sind sie nach Prozenten zu rechnen. Aber ein Prozent Verunreinigung kann den Schmelzpunkt um $3-10^{\circ}$ erniedrigen, und das deutlich zu beobachtende Schmelzintervall über einige hundert Grade ausdehnen, und Silikate mit $3 \%$ Verunreinigung zeigen in ganz entschiedener Weise das charakteristische Verhalten von Systemen aus $2 \mathrm{Kom}$ ponenten.

3. Experimentelle Bestimmung der der Beschickung zugeführten Wärmemenge. Eine quantitative Kenntnis der zugeführten Wärme ist offenbar wesentlich für eine genaue Schmelzkurve. Die Benutzung der Zeit als Mals der Wärme, was die Basis fast aller gewöhnlichen Schmelzpunktsmethoden bildet, ist als eine rohe Annäherung ${ }^{1}$ erkannt worden und würde in all den hier behandelten Fällen nicht genügt haben. Deswegen wurde ein genaueres Verfahren benutzt. Es beruht auf Anwendung des Kontrollelementes $C$ (Fig. 2), das eine Bestimmung des Temperaturunterschiedes von Ofen und Beschickung gestattet, wovon - und nicht von der Temperatur eines dieser Teile allein - der Wärmeflufs direkt abhängt. Dies Verfahren wird genauer in der folgenden Abhandlung S. 345 besprochen.

4. Einzelne Schmelzpunkte. Bei den meisten Bestim. mungen an schmelzenden Stoffen ist die Schmelztemperatur der einzige Gegenstand der Untersuchung. Diese Temperatur $\theta_{0}$ der Formel ist das obere Ende des Schmelzinterralles, der Punkt $X$ der Fig. 3 und 4. Die andere Charakteristik der Kurve, die Werte der spezifischen Wärme usw. sind dann meistenteils Fragen von geringerem Interesse. Nichtsdestoweniger sind anch in solchen Fällen die durch Verunreinigungen herrorgerufenen Komplikationen ebenso wichtig und störend wie in irgend einem anderen Falle. Denn in einem unreinen Stoff ist die gesuchte Schmelztemperatur nur ein Wert einer kontinuierlich veränderlichen Grộse. Bevor sie gemessen werden kann, mufs sie zuerst auf der geneigten Kurve festgelegt werden. Da sie aber tatsächlich an der Spitze des Schmelzintervalles liegt, und demnach beim Ende des Schmelzens, so fällt sie auf eine Stelle, wo die T'emperaturänderungen sehr unregelmälsig, verworren und unsicher zu deuten sind. Die hieraus

1 G. K. BunaEss, Methoden zur Bestimmung von Abkühlungskurven, Bull. Bur. Standards V, (1908), p. 223. - W. Rosenrain, Beobachtungen an Abkühlungskurven, Proe. Phys. Soc. Lóndon 21 (1908), 183. 
entstehenden Schwierigkeiten nehmen den Rest dieser Arbeit ein. Inzwischen zeigt ein Blick auf Fig. 1 die Schwierigkeit, die bei der Feststellung einzelner Schmelzpunkte auftreten kann.

III. Veränderliche Geschwindigkeit der Wärmezufuhr. Bei der Bestimmung des Schmelzpunktes einer ideal reinen Substanz allein ist die Art der Wärmezuführung ohne Bedeutung, solange sie nicht 0 oder unendlich grols wird. Für die genaue Bestimmung der Schmelzkurve eines unreinen Stoffes muls die Wärmezufuhr, wie gezeigt wurde, gut bekannt sein. Für die Bestimmung lediglich des Schmelzpunktes einer ziemlich unreinen Substanz erhält man dazwischen liegende Bedingungen; die Wärmezufuhr braucht dann nicht bekannt und auch nicht konstant zu sein, solange nur ibre Änderungen regelmälsig sind, d. h. der „Knick“ wird sich auf fast jeder glatten Kurve zeigen. Die Bestimmung eines Schmelzpunktes mit einer veränderlichen Wärmezuführung gibt jedoch oft Veranlassung zu einer sekundären Erscheinung, die so auffällig ist und so leicht zu Irrtümern führen kann, dals sie hier Erwähnung verdient. Dies tritt auf, wenn die Geschwindigkeit des Heizens, wie gewöhnlich, beinahe konstant gehalten wird. Sobald die Beschickung zu schmelzen beginnt, wird seine Temperatursteigerung unterbrochen, so dafs die andauernde Temperaturerhöhung des Ofens den Unterschied zwischen beiden erhöht; demnach bedingt die konstante Geschwindigkeit des Ofens notwendigerweise eine sehr veränderliche Geschwindigkeit der Wärmezuführung zur Beschickung. Das Ergebnis ist, dals der letzte Teil des Schmelzprozesses beschleunigt wird, wobei natürlich die Neigung zunimmt. Das Gleiche zeigt sich natürlich bei dem Ende einer Erstarrungskurve. Aber das Ende einer Schmelzkurve ist oben und das einer Erstarrungskurve unten. Hierdurch verlieren die beiden Arten Kurven alle Ähnlichkeit miteinander, indem die Schmelzkurve in ihrem oberen Teile mehr, die Erstarrungskurve weniger geneigt erscheint, als sie in Wirklichkeit sind. Der Beobachter, der konstante Verhältnisse aufrechtzuerhalten sucht, indem er sich dem Schmelz- oder Erstarrungspunkt mit derselben Geschwindigkeit nähert, und dann diese Geschwindigkeit im Ofen konstant erhält, kann leicht durch den kritischen Teil seiner Schmelzkurve fünf- oder zehnmal so schnell hindurchkommen als der Wirklichkeit entspricht. Wenn dagegen die anfängliche Geschwindigkeit durch Versuche so geregelt wird, dals sie zufriedenstellende Resultate beim Ende des Schmelzens gibt, so wird der einzige Nachteil der Zeitverlust bei der Aufnahme 
des ersten Teiles in einem langsamen Tempo sein, und dies ist bisweilen weniger nachteilig als der Ausweg, besondere Beobachtungen über die Ofentemperatur anzustellen und so durch Regulierung der Ofentemperatur den äufseren Temperaturgradienten konstant zu halten.

\section{Wärmeverteilung in der Beschickung.}

Die nächsten drei Ursachen der Neigung der Kurven umfassen das wichtigste praktische Problem bei Schmelzpunktsbestimmungen. Sie hängen von den Temperaturunterschieden in der Beschickung ab und könnten vermieden werden, wenn sorgfältiges und wirksames Rühren möglich wäre. Sie sind weniger schädlich bei der Bestimmung von Erstarrungs- als von Schmelzpunkten und wiederum weniger gefährlich bei Metallen, deren grofse thermische Leitfähigkeit z. T. das Fehlen der Rührung ersetzt. Diesen Ursachen ist es zuzuschreiben, dals unter sonst gleichen Verhältnissen der Schmelzpunkt eines Salzes gewöhnlich nicht mit derselben Genauigkeit bestimmt werden kann, wie der eines reinen Metalles oder wie der Erstarrungspunkt dieses Salzes. Die Fehler können jedoch sehr durch geeignete experimentelle Mafsnahmen vermindert werden.

IV. Der regelmäfsige und normale Temperaturgradient in der Beschickung. Der Fehler und die Unsicherheit, die aus dem Fehlen des Rührens sich ergeben, sind oft erheblich überschätzt worden. Die Beschickung als Ganzes mag grofse Temperaturunterschiede aufweisen, aber das Thermoelement verzeichnet sie nicht alle auf einmal. Es gibt nur die Temperatur des unmittelbar benachbarten Teiles an, und wenn dieser schmilzt, so zeigt es einen Knick in der Temperaturkurve. Gegen dieses kleine System von Element und umgebenden Material wirken die äufseren Teile der Beschickung in manchen Beziehungen wie eine gleiche Menge Fremdstoff, und noch dazu wie Fremdstoff, welcher besonders wegen seiner eigenen Wärmeabsorption störend ist. Ihr Einflufs kann mit einem für die hier beabsichtigten Zwecke hinreichendem Grade von Genauigk it untersucht werden durch die schon angewandte Methode $\because$ - sch nelzende Susstanz so zu behandeln als wenn sie von veränderlicher spezifischer Wärme wäre.

1. Ausdruck für die Temperaturverteilung in einer gewöhnlichen Beschickung. Wenn eine erhitzte Beschickung auf ihren Schmelzpunkt gebracht wird, so ist die Wärmezufuhr gewöhnlich konstant und dann wird die Geschwindigkeit der Temperaturerhöhung $d \theta / d t$ bald in allen Punkten gleich. Die sich er- 
gebende Temperaturverteilung in jedem Augenblick kann dann mit hinreichend grofser Annäherung in der folgenden Weise bestimmt werden: Nehmen wir zunächst an, dals der Körper eine Kugel mit dem Radius $R$ vorstelle, und dals der Wärmeflufs gänzlich in der Richtung der Radien verlaufe, und betrachten wir eine Kugelfläche $A$ in der Entfernung $r$ vom Mittelpunkt der Kugel. Ihre Fläche ist dann $4 \pi r^{2} ;$ das eingeschlossene Volumen $\frac{4 \pi r^{3}}{3}$. Der Wärmeflufs durch die Fläche kann dann auf zwei verschiedene Arten ausgedrückt werden: 1. direkt als Produkt von Fläche, Leitvermögen und Temperaturgefälle, d. b. gleich $4 \pi r^{2} K \frac{d \theta}{d t}$; 2. als Wärme, die ron dem eingeschlossenen Volumen absorbiert wird, oder als Produkt von Volumen, spezifischer Wärme des Volumens und Geschwindigkeit der Temperaturerhöhung, also gleich $\frac{4 \pi r^{3}}{3} S \frac{d \theta}{d t}$.

Setzt man diese beiden Ausdrücke gleich und integriert, indem man daran denkt, dals $\frac{d \theta}{d t}$ hier konstant ist, so erhält man

$$
[\Delta \theta]_{r}^{R}=\frac{S}{6 K} \frac{d \theta}{d t}\left(R^{2}-r^{2}\right) .
$$

Wenn die Beschickung ein unendlich langer Zylinder ist, so haben wir denselben Ausdruck, nur steht dann im Nenner auf der rechten Seite an Stelle von 6 die Zahl 4. Im allgemeinen können wir demnach sagen, dafs das Temperaturgefälle in einer gleichförmigen, festen, stetig erhitzten Beschickung sich einer Parabel nähert, und dafs die Temperaturdifferenz zwischen der Mitte und der Aufsenseite für Körper von gleicher Gestalt proportional ist der ersten Potenz der Erhitzungsgeschwindigkeit und dem Quadrat der Durchmesser.

Wenn wir setzen $\delta \theta=\Delta \theta$, dann ist $\delta t$ die Zeit, die für einen gegebenen Temperaturwert erforderlich ist, um von $R$ nach $r$ zu gelangen, d. h. es ist die Zeitverzögerung von $r$ hinter $R$. Diese ist gleich:

$$
\frac{S}{6 K}\left(R^{2}-r^{2}\right) .
$$

Sie ist unabhängig von der Geschwindigkeit der Temperaturerhöhung und ist demnach wichtig als eine Eigenschaft der Beschickung allein. 
2. Einflufs des Schmelzens auf die Temperaturverteilung. Bei einer wirklichen Schmelzpunktsbestimmung trifft eine fundamentale Hypothese, die bisher angenommen worden ist, nicht mehr zu. Die Temperaturerhöhung der Beschickung ist nach dem Beginn des Schmelzens nicht mehr regelmäfsig, was aber bei einer kleineren Beschickung nichts ausmacht da die Zeit zur Erreichung einer Gleichgewichtstemperaturverteilung relativ gering ist, und die Abweichung von dieser Verteilung in erster Annäherung vernachlässigt werden kann. Mathematisch wird dann das Problem einfach darauf zurückgeführt, dafs man den Einflufs einer Änderung der spezifischen Wärme feststellt, wenn die Geschwindigkeit der Wärmezuführung $\frac{d Q}{d t}$ konstant bleibt. Dies kann leicht in der folgenden Weise geschehen: $\operatorname{Da} d Q=S d \theta$, so ist

$$
d \theta=\begin{gathered}
d Q \\
d t
\end{gathered} \cdot \frac{1}{s} .
$$

Wenn demnach die spezitische Wärme $S$ um das $n$-fache zunimmt und $n S$ wird, so nimmt die Geschwindigkeit $\frac{d \theta}{d t}$ offenbar um das $n$-fache ab. Die Zeitrerzögerung (Gleich. 6) wächst um das n-fache. Die Temperaturverteilung kann durch Substitution von (7) in (5) gefunden werden. Das Resultat ist

$$
\Delta \theta_{\left[\frac{d Q}{d t}{ }_{\text {konsi }}\right]}=\frac{d Q}{d t} \cdot \frac{1}{6 K}\left(R^{2}-r^{2}\right)
$$

In diesem Ausdruck tritt die spezifische Wärme überhaupt nicht auf. Die Gleichgewichtstemperaturverteilung wird demnach durch eine Änderung der spezifischen Wärme nicht beeinflufst. Von diesen Ergebnissen einer Änderung der spezifischen Wärme ist die Zunahme der Zeitverzögerung von unmittelbarstem Interesse. Tritt sie bei konstanter Wärmezufuhr zu der ganzen Beschickung ein, so wird dadurch eine Verzögerung in der Zufuhr zum Mittelpunkt bedingt, und zwar ist diese dem Quadrat des Radius proportional. Die Formel drückt so in roher Weise quantitativ die auch sonst offenbare Tatsache aus, dals bei Beginn des Schmelzens die inneren Schichten abfallen, da die äulseren Schichten eine Zeitlang grofse Mengen von Wärme absorbieren, so dafs sehr wenig in das Innere eindringen kann. Drückt man dies durch die Temperatur aus, so wird 
die Verzögerung das $(n-1)$-fache der ursprünglichen Temperaturverzögerung betragen.

Dieses thrgebnis jedoch gilt, wie bereits gezeigt ist, streng nur für eine sehr kleine Beschickung, da es vollkommene Homogenität voraussetzt, die durch das Schmelzen selbst zerstört wird. In Wirklichkeit werden die äufseren Schichten, während der Körper schmilzt, eine höhere Temperatur und demnach eine grölsere spezifische Wärme haben als die inneren. Wie leicht gezeigt werden kann, wird hierdurch der Temperaturunterschied zwischen Mittelpunkt und Oberfläche vermindert und demnach auch die Zeitverzögerung, während eine Zunahme des Leitvermögens beim Schmelzen offenbar in derselben Richtung wirkt. Die Verzögerung im Mittelpunkt wird so in Wirklichkeit weniger schnell wachsen als das Quadrat des Fadius und für einen sehr grofsen Tiegel wird die angegebene Formel auch nicht näherungsweise zutreften.

Sobald die äufsere Schicht der Beschickung völlig geschmolzen ist, ändert sich der ganze Verlauf der Erscheinungen. Die virtuelle spezifische Wärme der Substanz während des Schmelzens ist gewöhnlich 50-100 mal so grofs als vorher oder nachher. Demnach hängt die Wärmekapazität des Ganzen eine Zeitlang fast vollständig von dem noch ungeschmolzenen Kern ab. In dem Mafse wie dieser sich vermindert, nimmt die relative Wärmezufuhr zu ihm zu, wodurch die Neigung des kritischen Endteiles der Kurve vermehrt wird. Auch ist die resultierende Abbiegung nicht, wie die oben (S. 315) beschriebene, von ungleichmälsiger Wärmezufuhr herrührende, nur eine allgemeine Vergrölserung der Steilheit des Endes. Sie ist vielmehr ein schnell beschleunigtes Wachsen, wodurch die Form der Kurve in dieser Gegend geändert wird. In grolsen Tiegeln verbirgt sie oft vollständig den Knick am Ende des Schmelzens, wofür dann ein vorzeitiger Knick $1-2^{0}$ tiefer eintritt, der zurückzuführen ist auf das starke Wachsen der Wärmezufuhr bevor die geschmolzene Schicht das Thermoelement überhaupt erreicht hat.

In Kürze hat demnach die unvermeidliche Wärmeverteilung in einer nicht gerührten Schmelze den folgenden Einfluls auf den Mittelpunkt, wo das Thermoelement sich befindet. Sie verzögert den normalen Temperaturanstieg beim Beginn des Schmelzens und gleicht z. T. den Verlust aus durch Beschleunigung derselben am Ende, wodurch die Neigung der Kurve vergrölsert wird, gerade an den Stellen, wo solche Zunahme am wenigsten wünschenswert ist. Dieser Einflufs wächst etwas weniger schnell als die Geschwindig- 
keit des Erhitzens und als das Quadrat des Durchmessers. Der Temperaturunterschied zwischen Mittelpunkt und Oberfläche bei konstanter Wärmezufuhr bleibt fast konstant für kleine Tiegel, nimmt aber während des Schmelzens ab für grofse Durchmesser und Geschwindigkeiten.

3. Grö se der Ablenkung. Diopsid, ein gut kristallisiertes Silikat, welches bei $1391^{\circ}$ schmilzt, zeigte eine Zeitverzögerung von $24 \pm 6$ Sekunden in einem Tiegel von $1 \mathrm{~cm}$ Radius, bevor das Schmelzen begann. Die Beschickung hatte die Form eines kurzen Zylinders. Die Schmelzwärme war hinreichend, um den festen Körper über $300^{\circ}$ zu erwärmen, aber sie war so verteilt, dals der Maximalwert der virtuellen spezifischen Wärme nur ungefähr $50 \mathrm{mal}$ so grofs war wie die wirkliche spezifische Wärme.

Wenn nun diese Substanz in einem Tiegel ron $1 \mathrm{~cm}$ Radius erhitzt wird mit konstanter Wärmezufuhr, die vor dem Schmelzen eine Temperaturerböhung von $8^{\circ}$ (pro Minute) gibt, so würde das Schmelzen ungefähr 35 Minuten dauern. Die Zeitverzögerung (Oberfläche bis Zentrum) würde nach Formel (6) 20 Minuten erreichen für eine Beschickung mit der maximalen spezifischen Wärme; wie aber der Versuch zeigte, betrug sie in diesem Falle nicht viel mehr als halb so viel. Selbst unter diesen Umständen aber dauert die Periode der beschleunigten Wärmezufuhr zum Mittelpunkt $10 \mathrm{Mi}$ nuten, so dafs das letzte Drittel (oder mehr) der Schmelzlinie abgelenkt werden wird. Dieses Drittel erstreckt sich über einen Intervall von $2-3^{\circ}$. Die entstehende Unsicherheit im Schmelzpunkt würde geringer sein, und demnach nicht sehr erheblich; aber bei einem Tiegel von $4 \mathrm{~cm}$ Durchmesser würde die Unsicherheit stark anwachsen.

Wenn der Tiegel $1 \mathrm{~cm}$ Durchmesser hat $(0.5 \mathrm{~cm}$ Radius), beträgt die Maximalverzögerung nach Formel (6) nur 5 Minuten, also $2 / 7$ des Schmelzintervalles, und das ganze Intervall, in dem beschleunigtes Ansteigen stattfindet, beträgt nicht über 0.5 Grad. Wenn dieser kleinere Tiegel jedoch eine Substanz von grölserer Reinheit enthält, z. B. Natriumchlorid, welches in einem Intervall von $1^{0}$ schmilzt, so kann die virtuelle spezifische Wärme $400 \mathrm{mal}$ so grofs werden wie die wahre, und das beschleunigte Intervall würde wieder ein Drittel oder mehr des Schmelzintervalles umfassen.

Die ablenkende Wirkung, die durch den pormalen Gradienten in der Beschickung auf der Schmelzkurve hervorgerufen wird, ist demnach praktisch nur bei grofsen Beschickungen ron Wichtigkeit, 
und wenn sie in diesen auch beträchtlich ist, so kann sie durch Verkleinerung der Gröfsenverhältnisse, die in der Praxis leicht herzustellen ist, so vermindert werden, dafs sie vernachlässigt werden kann. Die Ablenkung ist auch relativ am grölsten in den reinsten Substanzen, wo die Gesamtneigung am wenigsten schädlich wird.

Für Naphtalin war die Zeitverzögerung ungefähr dreimal so grofs wie für Diopsid. Für Metalle ist das Leitvermögen so grofs, dals eine Ablenkung der Art, wie soeben betrachtet wurde, in der Praxis fast immer zu vernachlässigen sein wird.

4. Die obigen Betrachtungen scheinen die folgenden Schlüsse für die Praxis zu rechtfertigen: 1. Für Bestimmungen von einzelnen Puukten ist es das Wesentlichste, das Intervall der beschleunigten Wärmezuführung zu vermindern. Dies bedingt die Anwendung von kleinen Tiegeln oder von geringen Geschwindigkeiten. Benutzt man diese, so können die Schwierigkeiten, die aus dem regulären Temperaturgradienten durch die Beschickung auftreten, im Vergleich mit den anderen Fehlerquellen zum Verschwinden gebracht werden. Die Erfordernisse bei einem gegebenen Fall hängen von der gewünschten Genauigkeit und der Reinheit der Substanz $\mathrm{ab}$; sie können für praktische Zwecke mit genügender Sicherheit festgestellt werden aus der Länge des Schmelzintervalles in Verbindung mit der Näherungsberechnung, die soeben für die Beziehung der Zeitverzögerung zum Radius in verschiedenen Substanzen mitgeteilt ist. 2. Zur Bestimmung der Form der ganzen Schmelzkurve muls die Zeitverzögerung klein gehalten werden und dies erfordert wieder einen engen Tiegel, aber nicht eine geringe Geschwindigkeit, aus Gründen, die in der folgenden Mitteilung S. 345 besprochen werden. 3. Ob Geschwindigkeit oder Radius oder beide zu vermindern sind, hängt hauptsächlich vom Apparat ab und von den Verbältnissen, unter denen der Experimentator arbeitet. Die ganze Zeit, die zur Beendigung des Schmelzens erforderlich ist, ändert sich beinahe wie $V$, die Geschwindigkeit des Erhitzens. Die Ablenkung ändert sich wie $R^{2} V$, aber $V$ ändert sich wie $G / R$, wo $G$ der äufsere Gradient ist. Demnach ändert sich die Ablenkung auch wie $G R$. Und demnach können für dieselbe Ablenkung durch Verminderung von $R, V$ und $G$ beide vermehrt werden. Wenn $R$ z. B. auf $1 / 3$ gebracht wird, so kann der Versuch in $1 / 9$ der Zeit beendigt werden, und der Einflufs des Wechsels oder der Unsicherheit in der Ofentemperatur kann gleichfalls um das Dreifache vermindert werden unter geringer Zunahme der Ablenkung. 
V. Unregelmäfsige Änderungen in der Wärmevertei. lung. Der vorstehende Abschnitt hat sich mit einem idealen Fall beschäftigt. . Ks wurde eine Beschickung angenommen, in der die Wärme gleichförmig bis zu einem unendlich kleinen Thermoelement fortschreitet, das sich genau in ihrem Mittelpunkt befindet. Natürlich sind diese Bedingungen niemals vollständig verwirklicht.

Ursachen der Unregelmälsigkeit können bedingt sein durch den Ofen, durch die Beschickung selbst oder durch ungenaues Zentrieren des Thermoelementes. Abgesehen hiervon besteht immer eine Tendenz dafür, dals das Schmeizen an einem Ende der Beschickung beginnt, entweder an der Oberfläche oder am Boden. Auf jeden Fall kommt die Schwierigkeit hauptsächlich daher, dafs die geschmolzene Fläche einige Teile des Thermoelementes vor den anderen erreicht, so dafs die Temperatur der Lötstelle von verschiedenen Teilen der Beschickung abhängt, von welchen einige geschmolzen, andere noch fest sind. Hierdurch wird der ideale scharfe Knick (X, Fig. 3) in ein allmähliches Ansteigen verwandelt, das nur unsicher gedeutet werden kann. Um dies zu vermeiden sind zwei Vorsichtsmafsregeln notwendig: Das Thermoelement selbst soll die Wärme möglichst wenig leiten, und das Schmelzen soll an der Seite beginuen und nicht am Ende. Dies erfordert ein Thermoelement ron feinem Draht und eine schmale aber nicht zu kurze Beschickung. Ungenaue Zentrierung des Thermoelementes ist offenbar ein geringerer Fehler. Der Einschlufs der Lötstelle in einem Porzellanrohr ist von diesem Gesichtspunkt aus nicht wünschenswert, da dann seine Temperatur von einem beträchtlichen Teil der Schmelze abbängt. Ein Widerstandsthermometer ist offenbar noch schlechter. In der Tat ist der Unterschied in der Schärfe zwischen Schmelzpunkten, die mit einem eingeschlossenen und einem ungeschützten Thermoelement bestimmt sind, sehr deutlich. Aber ein ungeschütztes Thermoelement aus Platindraht von $0.6 \mathrm{~mm}$ Durchmesser, das $8 \mathrm{~mm}$ in eine zylindrische Schmelze von $9 \times 14 \mathrm{~mm}$ eintaucht, gibt, wie wir gefunden haben (von $800^{\circ}$ aulwärts) ebenso scharfe Schmelzpunkte, wie man mit viel dünneren Drähten erzielen kann. Da düunere Drähte und schmälere und höhere Beschickungen leicht benutzt werden können, so scheint es nicht schwierig zu sein, den Einfluls unregelmärsiger Temperaturverteilung für solche Substanzen zu vermeiden, bei denen ein ungeschütztes Element benutzt werden kann. Einige Versuche zeigen, dafs bei niederen Temperaturen vielleicht 
Beschickungen erforderlich sind, die im Vergleich zur Höhe einen geringeren Durchmesser haben. Aber diese können bei niederen Temperaturen auch leicht benutzt werden.

VI. Wärmeleitung im Thermoelement. Die thermische Leitfähigkeit der meisten Substanzen ist viel geringer als die des metallischen Thermoelementes, so dafs der .Lötstelle oft Wärme durch die Drähte zugeleitet wird. Hierdurch wird eine allmähliche weitere Zunahme der Temperatur gegen das Ende des Schmelzens bedingt, wodurch die Neigung der Kurve vermebrt und auch eine Steigerung des Endknicks herbeigeführt wird. Die Wirkung wird nur wenig beeinflufst durch Änderung der Geschwindigkeit, da diese in der gleichen Weise auf die Wärme wirkt, einerlei ob sie nun durch die Beschickung oder durch den Draht einströmt. Dieser Einfluf's wächst mit dem Durchmesser der Beschickung, da hiermit die Schmelzdauer für denselben äufseren Gradienten wächst. $\mathrm{Er}$ wird vermindert mit zunehmender Dünnheit des Thermoelementes und durch tiefes Eintauchen in die Beschickung. Zur Vermeidung dieser Ursache einer Neigung sind demnach dieselben Bedingungen erforderlich wie für die vorhergehende, nämlich schmale und hohe Beschickungen und dünne Drähte des Thermoelementes. Der angeführte Versuch, welcher zeigt, dafs die eine Ursache der Neigung der Kurve leicht zu vermeiden ist, wird demnach das Gleiche auch für die andere Ursache beweisen. Andererseits gab ein $3 \mathrm{~cm}$ tief in einen Tiegel mit Natriumchlorid eingetauchtes, eingeschlossenes Thermoelement einen um $7^{\circ}$ zu hohen Schmelzpunkt. Dieser Fehler ist viel gröfser als der bei anderen höher schmelzenden Stoffen ${ }^{1}$ gefundene, aber er zeigt, dafs Schmelzpunktsmessungen mit geschütztem Thermoelement sehr vorsichtig gedeutet werden müssen. Eine hinreichende Schätzung des Fehlers, der aus der Wärmeleitung im Thermoelement entsteht, kann im allgemeinen leicht erzielt werden, indem man die Eintauchtiefe verändert. Bei Anwendung dieses Verfahrens auf $2.5 \mathrm{~g}$ Silikat ergab sich, dafs kein merklicher Fehler dieser Art bei einem 6-8 mm tief eingetauchtem Element festzustellen war, wodurch die Resultate bestätigt werden, die man durch Änderung der Dicke des Drahtes erhalten hat.

VII. Die Anwendung nackter Thermoelemente. Aus dem Vorhergehenden folgt klar, dafs in verschiedener Beziehung durch

1 Siehe: Isomorphismus $u$. therm. Eigenschaften von Feldspaten S. 23 bis 25; Diopsid und seine Beziehungen zu Calcium- und Magnesiummetasilikaten, S. $3-4$, l. c. 
Einführen des ungeschützten Thermoelementes in die Schmelze sehr grofse Vorteile sich ergeben. Ein Fehler kann aber hierdurch eingeführt werden, der durch die elekrische Leitfähigkeit der Beschickung selbst hervorgerufen wird, die nicht ganz zu vernachlässigen ist. Bei hohen Temperaturen leiten praktisch alle Stoffe die Elektrizität in gewissem Grade.

Die schwach leitende Beschickung wirkt wie eine Batterie von sehr hohem Widerstand die parallel mit der Lötstelle des Thermoelementes ${ }^{1}$ geschaltet ist. Der Einflufs einer solchen Batterie auf die Galvanometerablesung ist, wie man leicht sieht, im wesentlichen gleich $E_{s} \frac{T}{S}$, wo $\mathrm{E}_{s}$ eine E.M.K., $T$ der (sehr geringe) Widerstand des parallel geschalteten Teiles des Thermoelementes, $S$ der (sehr hohe) Widerstand des Nebenschlusses ist. Es folgt hieraus zuerst, dafs der Fehler durch das elektrische Leck mit der Tiefe des Eintauchens des Thermoelementes und der Dünne der Drähte wächst, und zweitens, dafs durch Eintauchen von Teilen eines Thermoelementes, die weit von der Lötstelle entfernt liegen, in eine Beschickung die Wirkung vergröfsert werden kann, so dafs hierdurch eine sehr empfindliche Prüfung auf diese Wirkung möglich ist. In der Tat braucht für diese Prüfung überhaupt keine Lötstelle vorhanden zu sein; ist eine vorhanden, so kann sie entweder auf Zimmertemperatur gehalten werden, oder ihre E.M.K. kann gemessen werden, indem man sie gleichzeitig zur Lötstelle zweier anderen Drähte macht, die nicht in die Beschickung eintauchen. Auf diese Weise ist gezeigt worden, dafs der auf diesen Einflufs zurückzuführende Fehler unter den von uns eingehaltenen Bedingungen nicht über $0.1-0.2^{\circ}$ hinaus geht, einerlei, ob es sich um Natriumchlorid bei $801^{\circ}$ oder um Diopsid bei $1391^{\circ}$ handelt. Es wurde auch für Natriumchlorid gezeigt, das man in einem Tiegel über einem Bunsenbrenner schmolz, dals die E.M.K. thermoelektrischer Natur ist und (in diesem Fall) vom Elektrolyten nach dem kälteren der beiden Drähte gerichtet ist. Demnach wächst der Fehler mit den in der Beschickung vorhandenen Temperaturdifferenzen. Die Konvektionsströme einer grofsen Beschickung zeigen sich häufig durch eine schwache Unstetigkeit des Galvanometers, und in einem Falle wurden die Störungen infolge des Leitvermögens der Schmelze um das 100 fache vergrölsert,

${ }^{1}$ Die Wirkung würde natürlich dieselbe sein, wenn sie irgend einen anderen Teil des Stromkreises schlösse. 
als man die Beschickung zu nahe an das obere Ende des Ofens brachte. ${ }^{1}$

Die Bedingungen, um den Fehler durch das Leck klein zu machen, sind demnach: Gleichförmigkeit der äufseren Temperatur, kleine Beschickung, kurze und dicke Drähte des Thermoelementes, soweit es eintaucht. Diese Forderungen widerstreiten z. T. den Bedingungen, die zur Verminderung der unter V. und VI. besprochenen Wirkungen dienen; aber mit Apparaten von den oben S. 322 angegebenen Grölsen werden alle diese Fehler auf $0.1-0.2^{\circ}$ oder weniger vermindert.

\section{Geringere Ursachen der Neigung der Kurve.}

VIII. Verunreinigung des Thermoelementes. Wenn der Ofen kontinuierlich und mehr oder weniger regelmäfsig während des Schmelzintervalles erhitzt wird, so wächst die Temperatur vom Ende des Ofens gegen die Beschickung fortwährend, und wenn das Thermoelement zwischen diesen beiden Stellen nicht homogen ist, so werden auch seine Anzeigen zunehmen. ${ }^{2}$ Im Falle eines stark verunreinigten Elementes bei $1400^{\circ}$ erwies sich die scheinbare Zunahme der Neigung selten grölser als $1^{0}$. Sie verschwindet im wesentlichen, wenn eine konstante Temperaturdifferenz zwischen Ofen und Beschickung aufrecht erhalten wird, sowie auch natürlich, wenn das Element in gutem Zustande ist; wenn also dieser Punkt auch niemals übersehen werden darf, so kann er doch auch niemals eine ernsthafte S.chwierigkeit bilden.

IX. Entmischung (Saigerung) in der Beschickung. Die Teile einer unreinen Beschickung, die zuletzt kristallisieren, können die Verunreinigungen angereichert enthalten, wodurch sie in der unmittelbaren Nähe des Thermoelementes dazu neigen werden, bei einer niedrigeren Temperatur zu schmelzen als an der Aufsenseite. Hierdurch würde die Neigung der beobachteten Kurve vergröfsert werden. Bisher sind wir noch nicht imstande gewesen, diesen Einflufs von den anderen begleitenden Ursachen der Neigung zu unterscheiden.

X. Strahlung durch die Beschickung. Wenn die schmelzende Beschickung diatherman ist, können direkte Strahlen von den Tiegel-

${ }^{1}$ Es ist demnach klar, dafs die angeführten Ergebnisse über den sehr kleinen aus der Leitfähigkeit der Schmelze entstehenden Fehler, bei steilen Gradienten (sehr hohen Temperaturen) eine ganz andere Gröfsenordnung annehmen können.

${ }^{2}$ Phys. Rev. 26 (1908), 535. 
wänden das Thermoelement erhitzen, wodurch eine Temperaturerhöhung verursacht wird, die am grölsten ist bei Beginn des Schmelzens, und die abhängt von den Änderungen der Ofentemperatur. Diese Wirkung konnte gleichfalls bisher nicht deutlich isoliert werden.

\section{Gegenwärtiger Stand des Problems.}

Fig. 4 A zeigt die typische Schmelzkurve eines ziemlich dünnflüssigen Silikats (Diopsid). Dal's die Neigung im unteren Teile dieser und ähnlicher Kurven auf die Substanz selbst (und demnach

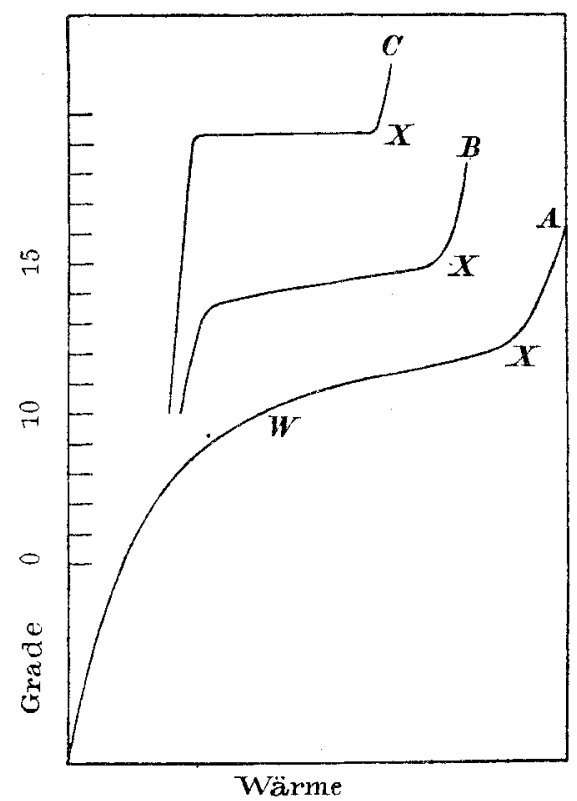

Fig. 4. Wirkliche Schmelzkurven $A$, Diopsid (Smp. $1391^{\circ}$ ); $B$, NaCl (Smp. $\left.801^{\circ}\right) ; C . \mathrm{Na}_{2} \mathrm{SO}_{4}\left(\mathrm{Smp} .885^{\circ}\right)$.

auf Verunreinigungen) zurückzuführen ist, kann leicht in der $t$ igenden Weise gezeigt werden: Die Neigung geht immer dem Schmelzen voran, wo dies auch stattfindet. Infolgedessen ist sie in der einen oder anderen Weise mit dem Schmelzen verknüpft und nicht auf den Ofen zurückzuführen, d. h. sie zeigt wirklich eine Wärmeabsorption an, die das Schmelzen begleitet. Wenn die Wärmeabsorption beginnt, so zeigt das Thermoelement in der Schmelze ungefähr $30^{\circ}$ weniger und ein nacktes Element direkt im Ofen aufserhalb der Beschickung gewöhnlich $25^{\circ}$ weniger an als dem schliefslich 
erhaltenem Schmelzpunkt entspricht. Demmach kann sich kein Teil der sehr kleinen Schmelze auf Schmelztemperatur oder in deren Nähe befinden, und demnach muls eine gewisse Wärmeabsorption durch Schmelzen wenigstens $20^{\circ}$ bereits unter dem Schmelzpunkt stattfinden. Auch ist die Neigung innerhalb der Experimentalfehler so, wie nach der bekannten Verunreinigung zu erwarten wäre. Durch Zusatz einer Verunreinigung kann sie in Silikaten vergröfsert und in ursprünglich reinen Substanzen hervorgerufen werden.

Der Verlauf der Kurve am oberen Ende von $W$ nach $X$ ist unverträglich mit den Gleichungen $1-4$ und kann nicht leicht gedeutet werden durch die Wirkung irgend einer anderen bekannten Verunreinigung. Es wurde der Versuch gemacht, die Ursache davon zu bestimmen. Änderungen in der Geschwindigkeit der Erhitzung, in der Eintauchtiefe des Thermoelementes, im Durchmesser der Drähte bewirkten keine wesentlıche Veränderung dieses Teiles der Kurve, woraus sich ergab, dafs weder die Ablenkung (IV) noch die Wärmeleiturıg durch das Thermoelement (VI) dafür herangezogen werden konnte.

Demnächst wurde eine leicht kristallisierende Substanz untersucht. Natriumchlorid (Erstarrungspunkt $\left.801^{\circ} \pm 1^{0}\right)^{1}$ gab die Kurve $B$ in Fig. 4, die dieselbe Eigentümlichkeit zeigt. Änderung der Geschwindigkeit des Erhitzens und der Grölse des Thermoelementes hatten keinen merklichen Einflufs. Der Erstarrungspunkt lag hier unterhalb des aus der Kurve bestimmten Schmelzpunktes. Demnach ist in diesem Fall die Gestalt des oberen Endes der Kurve auf die Temperaturmessung zurückzuführen und verursacht, dals der Schmelzpunkt etwas zu hoch gefunden wird. Wahrscheinlich gilt das Gleiche von den Silikaten, welche Kurven liefern wie $A$ in Fig. 4. In der Tat sind die Resultate, die man durch Zusatz verschiedener Mengen von Verunreinigungen zu einem dieser Silikate erhält, viel besser miteinander und mit Gleichung 1 in Übereinstimmung, wenn die Schmelzpunkte etwa $1^{0}$ niedriger angesetzt werden, als die Kurven zeigen.

Hierauf wurde Natriumsulfat (Schmelzpunkt $\left.885^{\circ} \pm 1^{9}\right)^{2}$ untersucht. Es resultierte die Kurve $C$ von Fig. 4. Das ganze Schmelzintervall betrug $0.1^{\circ}$ bei der einen, und $0.3^{\circ}$ bei einer anderen Beschickung, die wahrscheinlich be der Behandlung etwas verunreinigt war. Für eine so hohe Temperatur ist dies eine bemerkens-

1 Neue Bestimmung.

2 Neue Bestimmung. 
wert scharfe Kurve. Hält man dies mit dem Vorhergehenden zusammen, so zeigt sich, dals das falsche Ansteigen am Ende des Schmelzens vermieden werden kann. Ein Hinweis auf die Ursache mag in den physikalischen Unterschieden zwischen Natriumsulfat und Natriumchlorid bei ihren Schmelzpunkten gefunden werden. Eine weitere Untersuchung des Problemes scheint Apparate zu erfordern, die jetzt nicht zur Hand sind. Inzwischen ist der systematische Fehler für die Schmelzpunktsbestimmung in einem dünnflüssige Silikat auf etwa $1^{0}$ reduziert worden und die zufällige Änderung auf $0.5^{0}$, und diese Genauigkeit konnte erlangt werden mit aufserordentlich einfachen und bequemen Apparaten. Der Fehler im wahren Schmelzpunkt dieser Silikate, der auf Verunreinigungen zurückzuführen ist, wird (in unserem Fall) ebenso grols wie der experimentelle Fehler; seine Richtung ist die entgegengesetzte. Ein Versuch, gröfsere Genauigkeit zu erreichen, die wahrscheinlich nur durch bessere experimentelle Anordnung zu erzielen ist, schien bis jetzt nicht erforderlich zu sein.

Zusatz einer Verunreinigung zum Natriumsulfat von Fig. 4, C, gab die typische geneigte Kurve ron Fig. 3.

Hüttrer und Tammans ${ }^{1}$ entwickelten die Beziehung zwischen Verunreinigung und Neigung der Schmelzkurve, die hier in den Gleichungen 1-4 niedergelegt ist, und legten den Gedanken nahe, dafs die Form der Kurve ein zweckmälsiges Mittel sei, um nicht isomorphe Verunreinigungen $\mathrm{zu}$ bestimmen. Sie geben eine numerische Berechnung für die letzte Hälfte des Schmelzintervalles (oder die erste Hälfte des Erstarrungsintervalles), aber sie scheinen das Resultat übersehen zu haben, welches sie erhalten hätten, wenn sie die Berechnung auf ihrer Kurve weiter nach unten fortgesetzt hätten. Denn sie haben keine andere Erklärung für die gewöhnlich gefundene grolse Neigung an dieser Stelle als die Wärmeleitung im nackten Thermoelement, die sie als Hauptursache der Neigung bezeichnen. Ihre Ansicht über die wesentliche Ursache einer solchen Neigung ist demnach ganz entgegengesetzt dem Ergebnis, zu dem wir hier gelangt sind.

\section{Zusammenfassung.}

1. Die wirklich erhaltenen Schmelz- und Erstarrungspunktskurven sind fast immer geneigt, d. h. sie zeigen nicht die konstante

${ }^{1} \mathrm{~K}$. Hüttrer und G. Tamana, Über die Schmelzpunkte und Umwandlungspunkte einiger Salze, Z. anorg. Chem. 43 (1905), 218. 
Temperatur, die die elementare Theorie verlangt, sondern statt dessen ein Intervall, in dem die 'l'emperatur kontinuierlich steigt oder fällt.

2. Die erste Ursache der Neigung bei Schmelzkurven ist das durch Verunreinigungen bedingte unscharfe Schmelzen selbst. Der wahre Schmelzpunkt ist das obere Ende des geneigten Schmelzintervalles.

3. Die Schmelzhysteresis einiger sehr viskoser Stoffe (hauptsächlich Verbindungen von Bor und Silicium) ist gleichfalls eine gelegentliche, und dann wesentliche Ursache der Neigung.

Einige Ursachen der Neigung liegen in der experimentellen Bestimmung des Verhaltens schmelzender und erstarrender Stoffe.

4. Die Bestimmung einer Schmelzkurve umfalst notwendigerweise zwei Faktoren: Temperaturerhöhung und Wärmezufuhr; die letztere hängt von dem Temperaturunterschied zwischen Ofen und schmelzender Beschickung ab; wechselt dieser, so wird die Kurve in entschiedener Weise abgelenkt, was aber leicht korrigiert werden kann. Das gewöhnlichste und klarste Beispiel dafür ist der Fall, dafs man die Ofentemperatur kontinuierlich steigen oder fallen läfst, während die schmelzende oder erstarrende Substanz nahezu stationär bleibt.

5. Der Erstarrungspunkt, der beim (zeitlichen) Beginn des Intervalles liegt, wo die Temperaturverteilung in der Beschickung relativ gleichförmig ist, läfst sich leichter beobachten als der Schmelzpunkt, kann aber nicht benutzt werden für Stoffe mit ausgesprochener Unterkühlnng.

6. Der Schmelzpunkt, der am (zeitlichen) Ende des Intervalles liegt, kann leicht, wenn Rühren ausgeschlossen ist, Neigungen zeigen, die von ungleicher Temperaturverteilung herrühren: 1. infolge der unvermeidlichen Temperaturdifferenz zwischen dem Inneren und der Aufsenseite der Beschickung; dies stört bei grofsen Beschickungen, ist aber bei kleinen zu vernachlässigen; 2 . infolge der verschiedenen Unregelmälsigkeiten des Wärmeflusses, die gering sind, bei schmalen Beschickungen und dünnen Thermoelementen, kaum aber jemals $1-2^{0}$ überschreiten; 3. infolge der Fortführung von Würme entlang des Thermoelementes, was gleichfalls bei schmalen Beschickungen und dünnen Thermoelementen nur kleine Fehler verursacht, die in diesem Fall gewöhnlich zu vernachlässigen sind; diese letzte Unsicherheit kann aber bei eingeschlossenen Elementen auf mehrere Grade ansteigen. 
7. Elektrisches Leitvermögen du Schmelze ruft einen Fehter in der Ablesung der nackten Thermoelemente herror, der bei kleinen Beschickungen von Salzen gewöhnlich zu vernachlässigen ist.

8. Inhomogene Elemente geben falsche Ablesungen und bewirken auch ein Wachsen der Neigung der Kurve.

9. Entmischung (Saigerung) und Wärmedurchlässigkeit der Beschickung vermehren wahrscheinlich die Neigung.

10. Es sind Schmelzpunktsbestimmungen ausgeführt worden, oherhalb $800^{\circ}$, die miteinander auf $0.05^{\circ}$ übereinstimmen. In den meisten Fällen bleibt aber noch eine experimentelle Neigung von $0.5-1.5^{0}$ (bei hohen T'emperaturen) übrig, deren Ursache noch genau zu bestimmen ist.

Washington, D. C., Laboratory, Carnegie Institution, 15. Juli 1909.

Bei der Redaktion eingegangen am 19. Oktober 1910. 